\title{
Metallographische Mitteilungen aus dem Institut für anorganische Chemie der Universität Göttingen.
}

XLVI.

\section{Über die elektrische Leitfähigkeit der Legierungen.}

\author{
Von \\ W. GUERTLER.
}

\section{Der Zusammenhang zwischen der Konstitution und dem Tem- peraturkoeffizienten der Leitfähigkeit.}

Mit 10 Figtren im Text.

In einer früheren Arbeit (19) ${ }^{1}$ waren bezüglich des Zusammenhanges der elektrischen Leitfähigkeit der Legierungen mit ihrer Konstitution folgende beiden Resultate gewonnen worden:

1. die Leitfähigkeit der binären Metallegierungen, in denen keine Mischkrystalle auftreten, ist eine lineare Funktion der Volumkonzentration;

2. die Leitfähigkeit von metallischen Mischkrystallen ist stets aufserordentlich viel geringer als nach der Mischungsregel zu erwarten wäre.

Es waren damit Vermutungen ${ }^{2}$ im wesentlichen bestätigt worden, die Le Chatelier(10) schon im Jahre 1890 aussprach.

Weiterhin konnten die Gesetzmäfsigkeiten auf diejenigen Fälle ausgedehnt werden, in denen begrenzte Mischbarkeit der Komponenten im festen Zustande vorliegt, und auf diejenigen, in denen Verbindungen auftreten. Le Chatedings Vermutung, dals diese letzteren sich durch Maxima in den Leitfähigkeitskurven ausprägen

1 Alle Literaturangaben, S. 86-87.

${ }^{2}$ Ein Beweis der Richtigkeit seiner Vermutungen war ihm damals nicht möglich, da über die Konstitution und Struktur der von ihm betrachteten Legierungen (Blei-Zinn, Zink-Zinn, Cadmium-Blei, Cadmium-Zink, Cadmium-Zinn, Gold-Silber, Kupfer-Silber, Gold-Kupfer, Kupfer-Zinn, Gold-Zinn) gar keine oder nur unzuverlässige Untersuchungen vorlagen. 
würden, hat sich in der Folge nicht bestätigt. Vielmehr entspricht der Konzentration einer Verbindung auf der Leitfähigkeitskurve ein Knick. Stellt derselbe ein Maximum dar, wie bei den Verbindungen $\mathrm{Cu}_{3} \mathrm{Sn}, \mathrm{AuSn}$ und $\mathrm{Cu}_{2} \mathrm{Sb}$, oder auch ein Minimum wie bei $\mathrm{Cu}_{4} \mathrm{Sn}$, $\mathrm{AuSn}_{2}, \mathrm{Cu}_{3} \mathrm{Sb}$, so ist dies zufällig. Nur wenn eine Verbindung nach beiden Seiten bin bei Überschufs der einen oder der anderen Komponente Mischkrystalle zu bilden vermag, entspricht ihr auf der Leitfähigkeitskurve eine Spitze. Ganz allgemein ergab sich: Wird bei Vorhandensein von Metallverbindungen in binären Legierungen das Diagramm entsprechend diesen Verbindungen in Teildiagramme zerlegt, so prägen sich in diesen ebenso die Existenz von Mischkrystallen durch starke Erniedrigung der Leitfähigkeit und die Mischungsgrenzen unvollständiger Mischkrystallreihen durch Knicke in den Leitfähigkeitskurven aus, wie in Systemen zweier Metalle, die keine Verbindungen bilden.

Zur Vervollständigung der gegebenen Betrachtungen erschien es wünschenswert, den Einflufs der Temperatur auf den Verlauf der Leitfähigkeitskurven zu untersuchen. Ohne eine solche Erweiterung der Betrachtung blieb noch die Möglichkeit, dafs sich vielleicht die bei mischkrystallfreien Legierungen geradlinige, bei Mischkrystallreihen aber stark gekrümmte Gestalt der Leitfähigkeitskurven mit der Temperatur derart verschob, dal's die Stärke der Krümmung variierte und vielleicht jede Kurve die Form der geraden Linie bei einer bestimmten Temperatur einmal annahm. Die gefundenen Verschiedenheiten hätten sich dann als zufällige Folgen der willkürlich gewählten Versuchstemperaturen auffassen lassen.

Sollten also die gefundenen gesetzmälsigen Zusammenhänge zwischen Konstitution und Leitfähigkeit volle Gültigkeit behalten, so mufsten auch gewisse Zusammenhänge zwischen der Konstitution und dem Temperaturkoeffizienten erwartet werden.

Das vorhandene experimentelle Material, naturgemäfs schon weit dürftiger als das über die Leitfähigkeit selber, erwies sich gleichwohl zur Aufstellung erster allgemeiner Beziehungen durchaus als hinreichend.

Theoretische Betrachtungen über den Zusammenhang zwischen der Konstitution und dem Temperaturkoeffizienten fand ich nur bei LIEBEnow (18). Derselbe ging jedoch in seinen Theorien von einer falschen Basis aus, da er zwischen Mischkrystallen und Krystallgemengen überhaupt nicht unterschied, dieser Unterschied sich aber nunmehr als der wesentliche und entscheidende herausgestellt hat. 
Zwischen der Leitfähigkeit und ihrem Temperaturkoeffizienten stellten Matthiessen und Vogt(4) und Barus(8) Beziehungen auf, ohne jedoch dabei zu übereinstimmenden Resultaten zu gelangen.

\section{Vorbemerkungen.}

\section{Betreffend die Leitfähigkeit inhomogener Leiter.}

Es war in der vorangehenden Arbeit gezeigt worden, dals in mischkrystallfreien Legierungen die Leitfähigkeit annähernd nach der Mischungsregel berechenbar ist aus den Leitfähigkeiten der Komponenten und ihren relativen Volumenmengen. Es entsteht jedoch nun die Frage, ob der Widerstand oder die Leitfähigkeit eine lineare Funktion der Volumkonzentration sein soll. Da diese beiden Funktionen in reziprokem Verhältnis zueinander stehen, so mufs immer die eine durch eine Kurve ausgedrückt sein, wenn die andere geradlinig angenommen wird. Man kann also bei der Mittelwertsberechnung je nach dem Ausgangsansatz sowohl für den Widerstand $W_{m}$ wie für die Leitfähigkeit $\varkappa_{m}$ entweder eine gerade Linie oder eine Kurve erhalten. Die Abweichung beider Linien voneinander hängt von der Differenz der Leitfähigkeiten der beiden Komponenten ab.

Die Theorie der Stromverteilung in inhomogenen Leitern lehrt, dafs bei Abwesenheit aller sekundären Erscheinungen (Thermokräfte usw.), weder die Leitfähigkeit noch der Widerstand eine lineare Funktion der Volumenkonzentration sein kann, sondern mittlere Werte erhalten werden, je nach der räumlichen Anordnung der beiden Krystallarten in der Legierung. In der Tat schliefsen sich manche Legierungen der Linearität des Widerstandes, manche der der Leitfähigkeit näher an, und es ist im folgenden grölstenteils mit Matrhiessen die Leitfähigkeit, in einigen Fällen jedoch auch der Widerstand als linear angenommen worden.

\section{Betreffend den Temperaturkoeffizienten.}

Die Abhängigkeit des spezifischen Widerstandes von der Temperatur findet sich meist gegeben durch eine Gleichung von folgender Form:

$$
W=W_{0}\left(1+\alpha t+\beta t^{2}+\ldots\right)
$$

( $W=$ Widerstand, $W_{0}$ derselbe bei $0^{0}, t=$ Temperatur, $\alpha$ und $\beta$ Konstanten.) Die analoge Gleichung für die Leitfähigkeit ergibt sich daraus durch Reihenentwickelung: 


$$
\begin{gathered}
-61- \\
x=x_{0}\left(1-\alpha t+\left[\alpha^{2}-\dot{\beta}\right] t^{2}+\ldots\right) .
\end{gathered}
$$

Die kubischen und höheren Glieder lassen sich hier stets unbedenklich vernachlässigen, nicht aber die quadratischen. Weder $\beta$ in Gl. (1) noch viel weniger $\left(\alpha^{2}-\beta\right)$ in Gl. (2) ist exakt gleich $0 \mathrm{zu}$ setzen. Die Abhängigkeit weder der Leitfähigkeit noch auch meistens des Widerstandes von der Temperatur ist linear. Es erscheint deshalb ratsam, den Vergleichen des Temperaturkoeffizienten einen Ausdruck zugrunde zu legen, der $\alpha$ und $\beta$ gleichzeitig umfarst. Greifen wir darum ein beliebiges Temperaturintervall, etwa das von $0-100^{\circ}$, heraus, so wird:

$$
\frac{W_{100}-W_{0}}{W_{0}}=\alpha \cdot 100+\beta \cdot 100^{2} .
$$

Setzen wir diesen Ausdruck $=\frac{P_{z}}{100}$, so ist $P_{z}$ die prozentische $\mathrm{Zu}-$ nahme des Widerstandes durch Erhöhung der Temperatur von 0 auf $100^{\circ}$ oder der Leitfähigkeit durch Erniedrigung derselben von 100 auf $0^{\circ}$. Es ist also

$$
\frac{W_{100}-W_{0}}{W_{0}}=\frac{x_{0}-x_{100}}{x_{100}}=\frac{P_{z}}{100} .
$$

Mit demselben Recht liefse sich aber auch die prozentische Abnahme der Leitfähigkeit, $P_{a}$, durch Erhöhung der Temperatur von 0 auf $100^{\circ}$, den Rechnungen zugrunde legen und man hätte dann die Gleichung:

$$
\frac{x_{0}-x_{100}}{x_{0}}=\frac{W_{100}-W_{0}}{W_{100}}=\frac{P}{100} .
$$

Der Zusammenhang zwischen $P_{z}$ und $P_{a}$ ergibt sich aus Gl. (3), (4) und (1 a):

$$
\begin{gathered}
\frac{P_{a}}{P_{z}}=\frac{x_{100}}{x_{0}}=\frac{W_{0}}{W_{100}}=\frac{1}{1+100(\alpha+100 \beta)}=\frac{1}{1+\frac{P_{z}}{100}} \\
P_{a}=\frac{100 P_{z}}{100+P_{z}} .
\end{gathered}
$$

Es zeigt sich hieraus, dafs $P_{a}$ und $P_{z}$ im allgemeinen einander nicht proportional sind. Nur in den - allerdings weitaus meisten Fällen, dafs $P_{z}$ nur wenige Einheiten beträgt, wird die Proportionalität der beiden Gröfsen angenähert erreicht. 
Dem Ausdruck $P_{z}$ sehr verwandt ist der Differentialquotient des Widerstandes nach der Temperatur. Derselbe folgt aus Gl. (1):

$$
\frac{d W}{d t}=\alpha+2 \beta t \text {. }
$$

Er gibt die Tangente der $W$-t-Kurve für eine bestimmte Temperatur ganz ähnlich wie $P_{z}$ gemäfs Gl. (1a) die mittlere Tangente von 0 bis $100^{\circ}$ darstellt. In dieser Gestalt findet sich der Temperaturkoeffizient gleichfalls gelegentlich in der Literatur gegeben und ist dort meist, im Gegensatz zu der hier gewählten Bezeichnungsweise, mit $\alpha$ bezeichnet.

Es ist nun im folgenden im Anschlufs an MatThiEssen $P_{a}$ den Vergleichen zugrunde gelegt und kurz mit $P$ bezeichnet.

Die von den verschiedenen Forschern gegebenen Daten sind in der Weise verwertet, dafs aus den mitgeteilten Tabellen, Figuren oder Gleichungen die Werte $W_{0}$ und $W_{100}$ entnommen und daraus nach Gl. (4) $P_{a}$ berechnet ist.

Für die reinen Metalle gilt angenähert das Gesetz, dafs der spezifische Widerstand derselben beim absoluten Nullpunkt der Temperatur verschwindet und proportional der absoluten Temperatur wächst. In diesem Falle ist

$$
\begin{gathered}
\beta=0 \\
\alpha=0.0036630
\end{gathered}
$$

somit:

$$
P_{z}=\alpha \cdot 10^{4}=36.63
$$

und nach Gl. (5):

$$
P_{a}=26.80965 .
$$

Diesen idealen Werten kommen die an reinen Metallen beobachteten, wie sie in Tabelle 1 zusammengestellt sind, nur mehr oder weniger nahe. Sie sind stets zu grols. $P_{a}$ schwankt im allgemeinen von 27 bis $31, P_{z}$ von 37 bis 45 . (Nur bei den magnetischen Metallen, Eisen und Nickel, wird die Abweichung weit erheblicher.) Die Abweichung der Angaben der einzelnen Beobachter voneinander sind vielfach grölser als die Differenzen zwischen ihren Angaben für die verschiedenen Metalle. Noch unaufgeklärte Umstände rufen hier grolse Unsicherheit der Melsresultate hervor. Gröfsere Regelmälsigkeit dürfen wir also im folgenden auch bei den Legierungen nicht erwarten. 
Tabelle 1.

Temperaturkoeffizient der reinen Metalle.

\begin{tabular}{|c|c|c|c|}
\hline Metall & $P_{a}$ & $P_{z}$ & Gewährsmann \\
\hline $\mathrm{Pb}$ & $\left\{\begin{array}{l}28.6 \\
29.9\end{array}\right.$ & $\begin{array}{l}40.1 \\
42.7\end{array}$ & $\begin{array}{l}\text { Jaeger und Disselhorst } \\
\text { Lorenz }\end{array}$ \\
\hline Sn & $\left\{\begin{array}{l}30.2 \\
31.1 \\
30.0 \\
29.1\end{array}\right.$ & $\begin{array}{l}43.3 \\
45.2 \\
42.9 \\
41.1\end{array}$ & $\begin{array}{c}\text { Dewar und Fleming } \\
\text { Jaeger und Disselhorst } \\
\text { Ihle }\end{array}$ \\
\hline $\mathrm{Bi}$ & $\left\{\begin{array}{l}32.2 \\
29.7 \\
34.6\end{array}\right.$ & $\begin{array}{l}47.5 \\
42.2 \\
52.9\end{array}$ & $\begin{array}{l}\text { Lorenz } \\
\text { Jaeger und Disselhorst } \\
\text { Dewar und Fleming }\end{array}$ \\
\hline $\mathrm{Zn}$ & $\left\{\begin{array}{l}27.1 \\
32.8\end{array}\right.$ & $\begin{array}{l}37.2 \\
48.8\end{array}$ & $\begin{array}{l}\text { Jaeger und Disselhorst } \\
\text { Dewar und Fleming }\end{array}$ \\
\hline $\mathrm{Cd}$ & 29.3 & 41.5 & Lorenz \\
\hline $\mathrm{Cu}$ & $\left\{\begin{array}{l}30.3 \\
28.4\end{array}\right.$ & $\begin{array}{l}43.5 \\
39.7\end{array}$ & $\begin{array}{l}\text { Dewar und Fleming } \\
\text { Jaeger und Disselhorst }\end{array}$ \\
\hline $\mathrm{Ag}$ & $\left\{\begin{array}{l}29.3 \\
27.4\end{array}\right.$ & $\begin{array}{l}41.5 \\
37.8\end{array}$ & $\begin{array}{l}\text { Dewar und Fleming } \\
\text { Jaeger und Disselhorst }\end{array}$ \\
\hline $\mathrm{Au}$ & $\left\{\begin{array}{l}27.9 \\
28.9 \\
25.8\end{array}\right.$ & $\begin{array}{l}38.7 \\
40.7 \\
34.9\end{array}$ & $\begin{array}{l}\text { Dewar und Fleming } \\
\text { Ihle } \\
\text { Jaeger und Disselhorst }\end{array}$ \\
\hline $\mathrm{Pt}$ & $\left\{\begin{array}{l}26.2 \\
26.5\end{array}\right.$ & $\begin{array}{l}35.5 \\
36.1\end{array}$ & $\begin{array}{l}\text { Dewar und Fleming } \\
\text { Jaeger und Disselhorst }\end{array}$ \\
\hline $\mathrm{Fe}$ & 51.0 & 104.1 & Dewar und Fleming \\
\hline $\mathrm{Ni}$ & 38.8 & 63.4 & Fleming \\
\hline
\end{tabular}

\section{Legierungen ohne Mischkrystalle.}

Wie in der ersten Arbeit dargelegt wurde, ist bei mischkrystallfreien Legierungen die Leitfähigkeit angenähert linear von der Volumenkonzentration abhängig. Mit der gleichen Annäherung kann in den fünf hierher gehörigen Fällen (die Legierungen $\mathrm{Cd}-\mathrm{Zn}$, $\mathrm{Cd}-\mathrm{Sn}, \mathrm{Cd}-\mathrm{Pb}, \mathrm{Zn}-\mathrm{Sn}, \mathrm{Sn}-\mathrm{Pb}$ ) aber auch der Widerstand linear angesehen werden (cf. S. 60), zumal die Differenz der Leitfähig. keiten der betreffenden vier Metalle nicht sehr erheblich ist. Dieses letztere wollen wir der Einfachheit halber annehmen, da obnehin der $Z$ weck dieser Arbeit nur der ist, erste Näherungsregeln für die Werte des Widerstandes zu gewinnen. Die lineare Ab- 
Tabelle 2.

Gemischte Reihe nach Matthiessen und Voat.

\begin{tabular}{c|c|c}
\hline $\begin{array}{c}\text { Legierungen von der } \\
\text { Zusammensetzung }\end{array}$ & $P$ & $\alpha$ \\
\hline $\mathrm{Sn}_{6} \mathrm{~Pb}$ & 30.18 & 0.00389 \\
$\mathrm{Sn}_{4} \mathrm{Cd}$ & 28.89 & 0.00408 \\
$\mathrm{Sn}_{2} \mathrm{Zn}$ & 30.12 & 0.00388 \\
$\mathrm{PbSn}$ & 29.41 & 0.00379 \\
$\mathrm{ZnCd}_{2}$ & 29.86 & 0.00374 \\
$\mathrm{SnCd}_{4}$ & 29.08 & 0.00385 \\
$\mathrm{CdPb}_{6}$ & 27.74 & 0.00351
\end{tabular}

hängigkeit kann nur dann eine allgemeine von der Temperatur unabhängige Eigenschaft sein, wenn die Temperaturkoeffizienten $P$ aller Legierungen einer solchen mischkrystallfreien Reihe konstant und den entsprechenden Werten der reinen Komponenten gleich sind. Diese Forderung, die schon Matthiessen und Vogt(4) aufstellten, bestätigten sie durch eine Reihe von Messungen, welche in Tabelle 2 zusammengestellt sind. Da der quadratische Koeffizient $\beta$ in diesen Legierungen zufällig nahezu vernachlässigt werden darf, so ist hier auch $a$ annähernd vergleichbar und darum in Tabelle 2 in der letzten Spalte hinzugefügt. Ein Vergleich mit Tabelle 1 zeigt, dafs die Schwankungen der Werte sogar weit geringer sind als die der an reinen Metallen gemessenen Zahlen.

Zur Bestätigung der Resultate Matrhiessens lälst sich eine Arbeit von WEBER (7) heranziehen. Die aus den mitgeteilten Daten berechneten Werte ${ }^{1}$ von $P$ sind in Tabelle 3 zusammengestellt und

Tabelle 3.

Blei-Zinn nach Weber.

\begin{tabular}{c|c}
\hline Gewichts-\% Blei & $P$ \\
\hline 0 & 30.0 \\
10.06 & 29.3 \\
21.65 & 29.1 \\
33.47 & 28.7 \\
40.27 & 29.0 \\
50.14 & 28.1 \\
60.01 & 27.4 \\
100 & 28.6
\end{tabular}

${ }^{1}$ Aus den sehr zahlreichen Daten wurden nach der Methode der kleinsten Quadrate Gleichungen gewonnen mit deren Hilfe $W_{0}$ und $W_{100}$ intrapoliert werden konnten. 
in Figur 2 (S. 67) eingetragen. Es zeigt sich, dals sie sich innerhalb der Beobachtungsfehler (im Gegensatz zu den anderen Kurven in derselben Figur) zu einer geraden Linie zusammenfassen lassen.

Verwerten wir wieder, wie in der ersten Arbeit, die Erkenntnis, dals die hier in Rede stehenden Legierungen alle frei sind von Mischkrystallen, so gelangen wir zu dem Satz:

Der Temperaturkoeffizient der mischkrystallfreien Legierungen ist dem der reinen Metalle gleich.

Findet man umgekehrt den Temperaturkoeffizienten j. einer Legierung gleich 27 oder gröfser, so entbält dieselbe keine Mischkrystalle (magnetische Legierungen ausgenommen).

Ferner ergibt sich, dals der Widerstand all dieser Legierungen ebenso wie der der reinen Metalle beim absoluten Nullpunkt verschwinden mufs. Das ist durchaus verständlich, da der Widerstand jedes einzelnen Krystalles der beiden durcheinander liegenden Arten verschwindet. Stellt man sich ein Koordinatensystem her mit den Achsen des Widerstandes (vertikal) der Konzentration (horizontal) und der absoluten Temperatur (normal zur Zeichenebene), so ergibt sich eine flache Schraubenfläche von

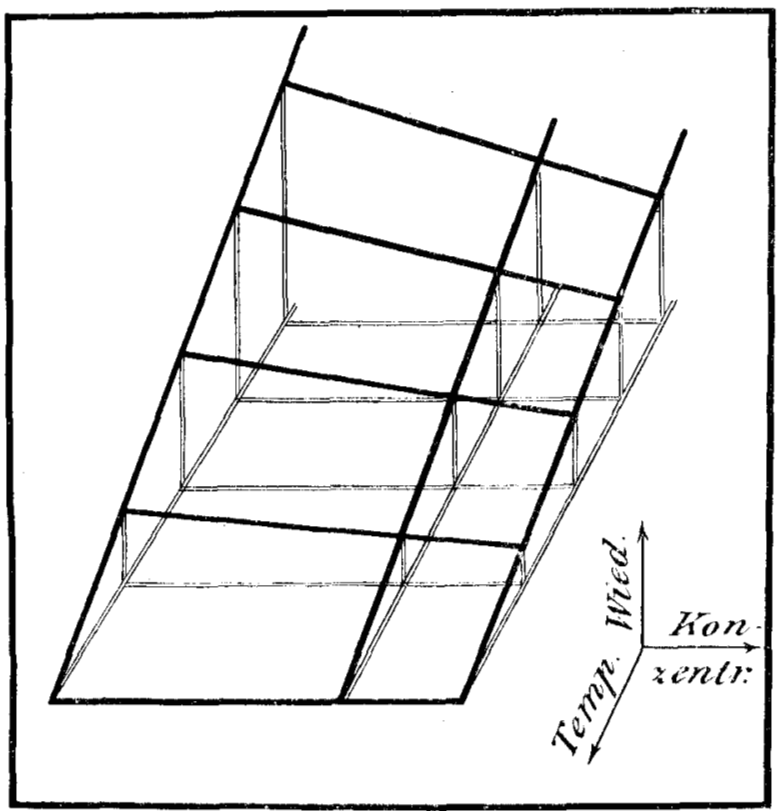

Fig. 1. Spez. Widerstand bei binären Gemengen. 
etwa der Gestalt, wie sie in Fig. 1 dargestellt ist. Vereinfachend ist dort die Abhängigkeit des Widerstandes sowohl von der Konzentration wie von der Temperatur geradlinig gezeichnet worden. Das Auftreten eines quadratischen Gliedes in der $W$ - $t$-Gleichung (Gl. (1) erzeugt eine gröfsere oder geringere Krümmung der $W$ - $t$-Geraden nach oben. Tritt eine solche bei den reinen Komponenten auf, so müssen wir sie ebenso bei ihren Legierungen wiederfinden.

\section{Legierungen mit Mischkrystallen.}

\section{Einige Kurven.}

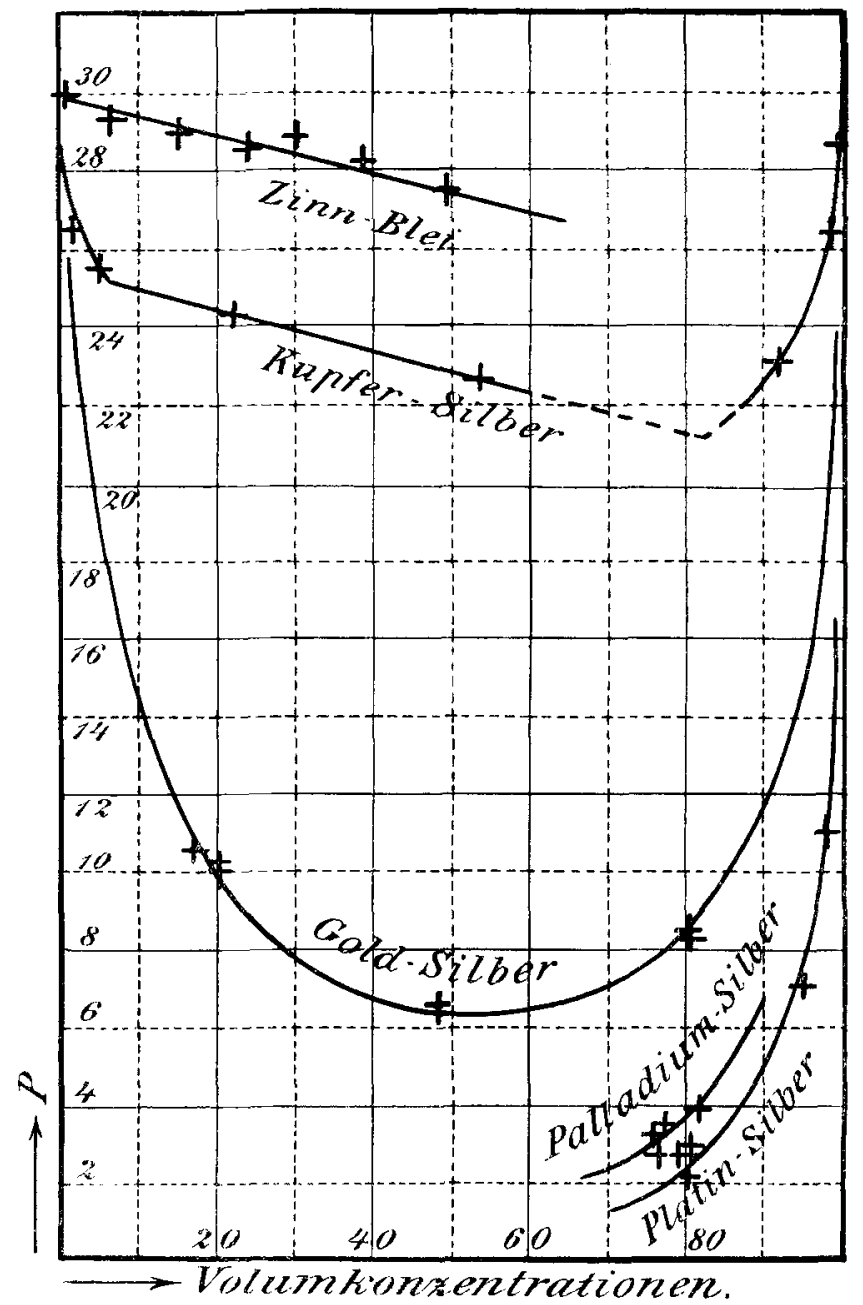

Fig. 2. Blei-Zimm und verschiedene Silberlegierungen. 
An Legierungen, welche Mischkrystalle enthalten, sind zahlreiche Messungen des Temperaturkoeffizienten von verschiedenen Forschern

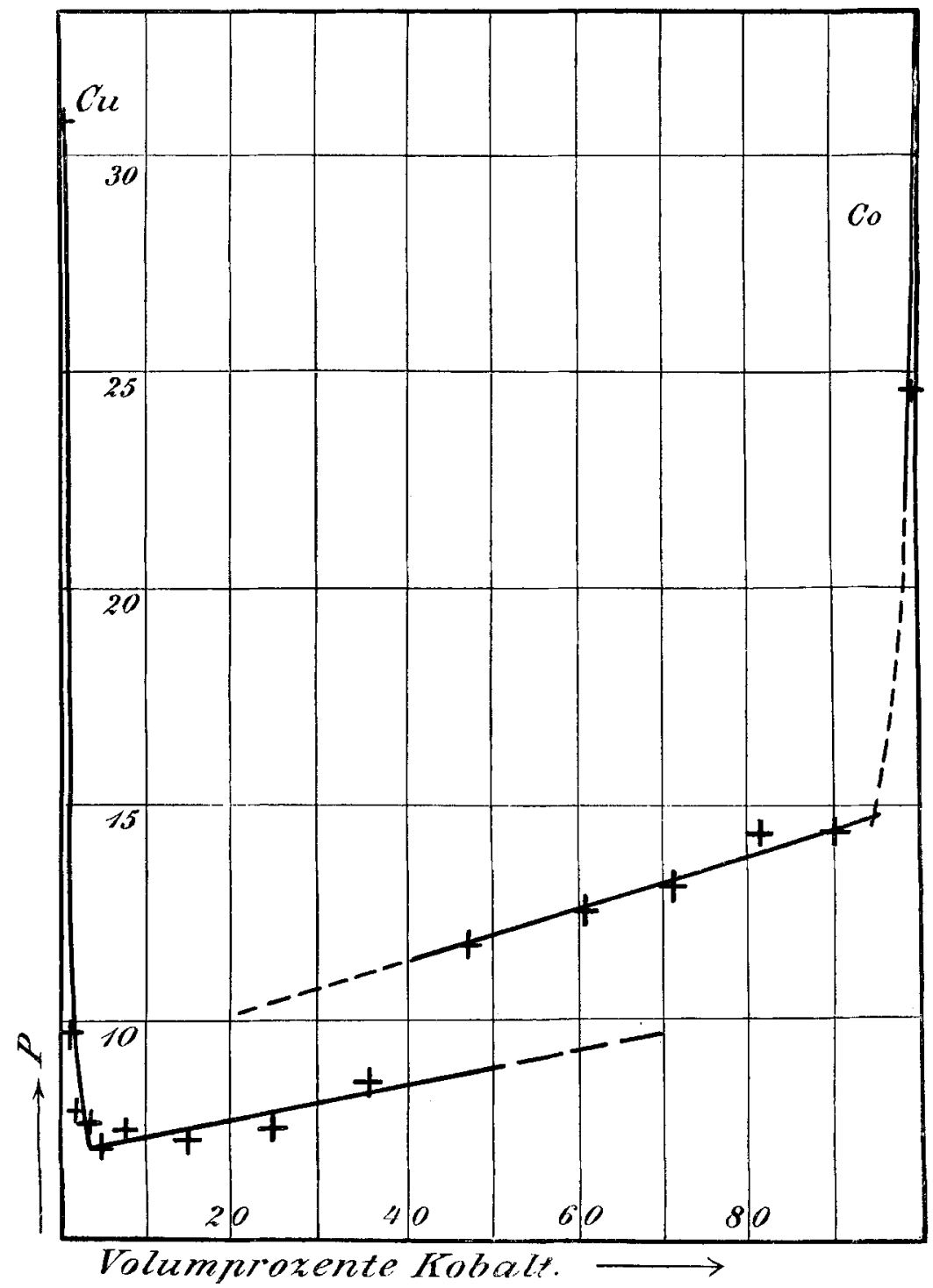

Fig. 3. Kupfer-Kobalt nach Reichuardt.

ausgeführt, doch fehlt es zurzeit noch an zusammenhängenden Untersuchungen vollständiger Reihen. Bislang lälst sich nur der Verlauf weniger Kurven mit einiger Genauigkeit festlegen.

Betreffend einige Legierungen von Silber mit Gold, Kupfer, 
Platin und Palladium verdanken wir Matrhiessen und Vogt (4) und Dewar und Fleming (11)(12) Daten, die in Fig. 2 eingetragen sind. Zunächst fällt auf, dafs wir bei den Kurven dieser Silberlegierungen im Gegensatz zu der schon oben besprochenen Zinn-Blei-Kurve in derselben Figur der gleichen Gestalt wieder begegnen, welche wir früher bei den Leitfähigkeitskurven derselben Legierungen als typisch bei Gegenwart von Mischkrystallen erkannten (cf. l. c. S. 403, 404). Die Werte der Temperaturkoeffizienten fallen beiderseits von den reinen Metallen ausgehend aufserordentlich steil ab. In der Kupfer-Silber-Kurve prägt sich die Mischungslücke durch ein geradliniges Mittelstück aus, und die Vermutung drängt sich auf, dafs auch hier wieder die beiden Knicke den Konzentrationen der gesättigten Mischkrystalle entsprechen werden. ${ }^{1}$

Fig. 3 zeigt die P-Kurve der Kupfer-Kobalt-Legierungen nach der Untersuchung von ReichHARDt (16). Wiederum finden wir hier für den Temperaturkoeffizienten ganz die gleiche Abhängigkeit von

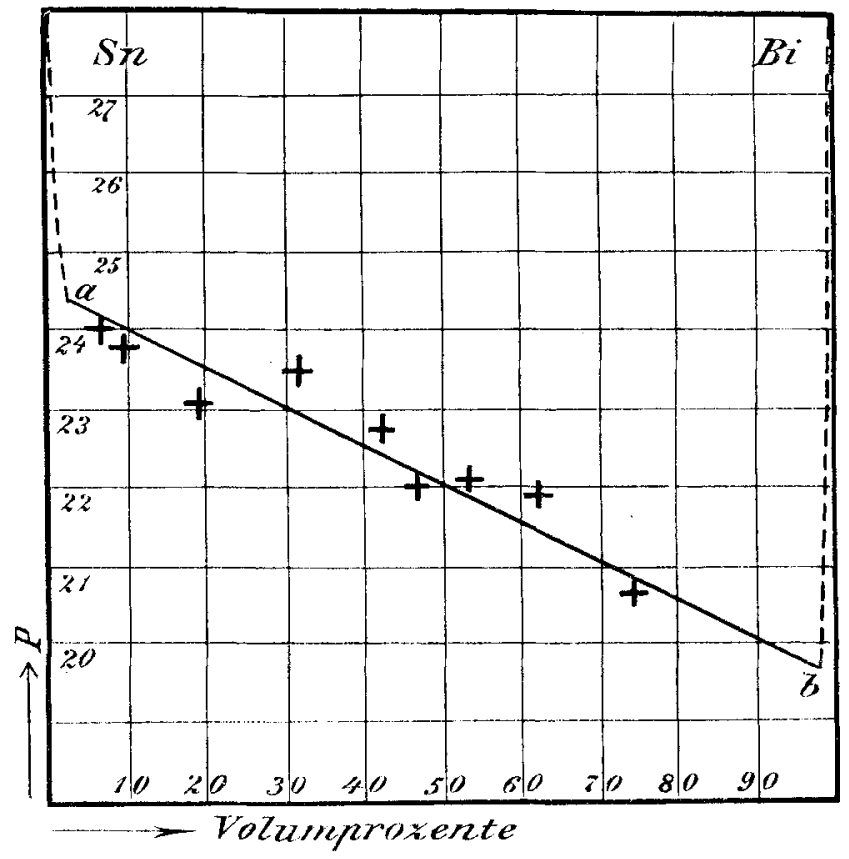

Fig. 4. Zimn-Wismut nach Weber.

${ }^{1}$ Diese Konzentrationen ergeben sich hier vorerst anders als aus der Leitfähigkeitskurve, doch ist dies bei der mangelnden Schärfe und geringen Anzahl der Untersuchungen nicht verwunderlich. 
der Konzentration wie in der früheren Untersuchung (S. 405) für die Leitfähigkeit selber. Auch die Diskontinuität in der Mitte des geradlinigen Mittelstückes findet sich hier wie dort und hat die gleiche Erklärung. Der grolsen Mischungslücke entspricht also auch hier wieder ein geradliniges Mittelstück. Die Existenz von Mischkrystallen auf beiden Seiten in dem Bereiche, wie sie die Knicke in der $H$. und der $P$-Kurve übereinstimmend erwarten lassen, hat inzwischen eine eingehende Untersuchung von R. SAHMEN im hiesigen Institut bestätigt.

Dasselbe Bild bieten die P-Werte der Zinn-Wismut-Legierungen (Fig. 4) nach WEBER (7). Die Mischungslücke im krystallisierten Zustande ist hier noch gröfser. Die beobachteten Daten fallen alle innerhalb derselben und liefern daher eine gerade Linie. Die Verlängerung dieser erreicht die Achsen der reinen Metalle bei aufserordentlich viel geringeren $P$-Werten als den reinen Metallen selber, Zinn und Wismut, zukommen. Es mufs daher nahe den Achsen der beiden reinen Komponenten ein steiler Anstieg der $P$-Kurve stattfinden. Einen solchen Anstieg zeigten schon die $x$-Werte dieser Legierungen (l. c. S. 409, 410), und es ist schon dort die Existenz von Mischkrystallen, wenn auch nur innerhalb geringer Konzen-

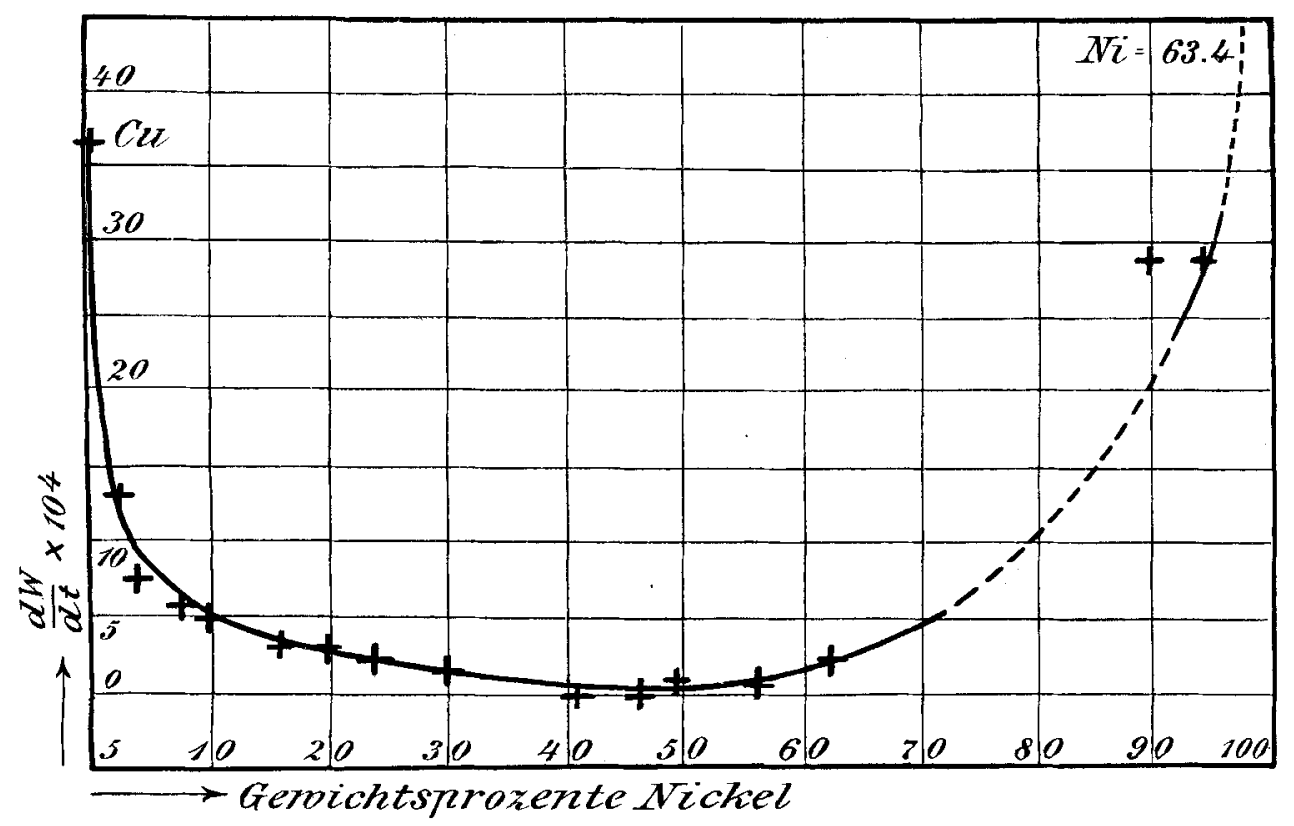

Fig. 5. Kupfer-Nickel nach Frusaner und Lindeck. 


\section{$-70-$}

trationsgebiete, angenommen und (S. 411) teilweise nacbgewiesen worden.

Ferner geben Fuussner und Lindeck (14) die Temperaturkoeffizienten einer Reihe Kupfer-Nickel- und Kupfer-Mangan-Legierungen,

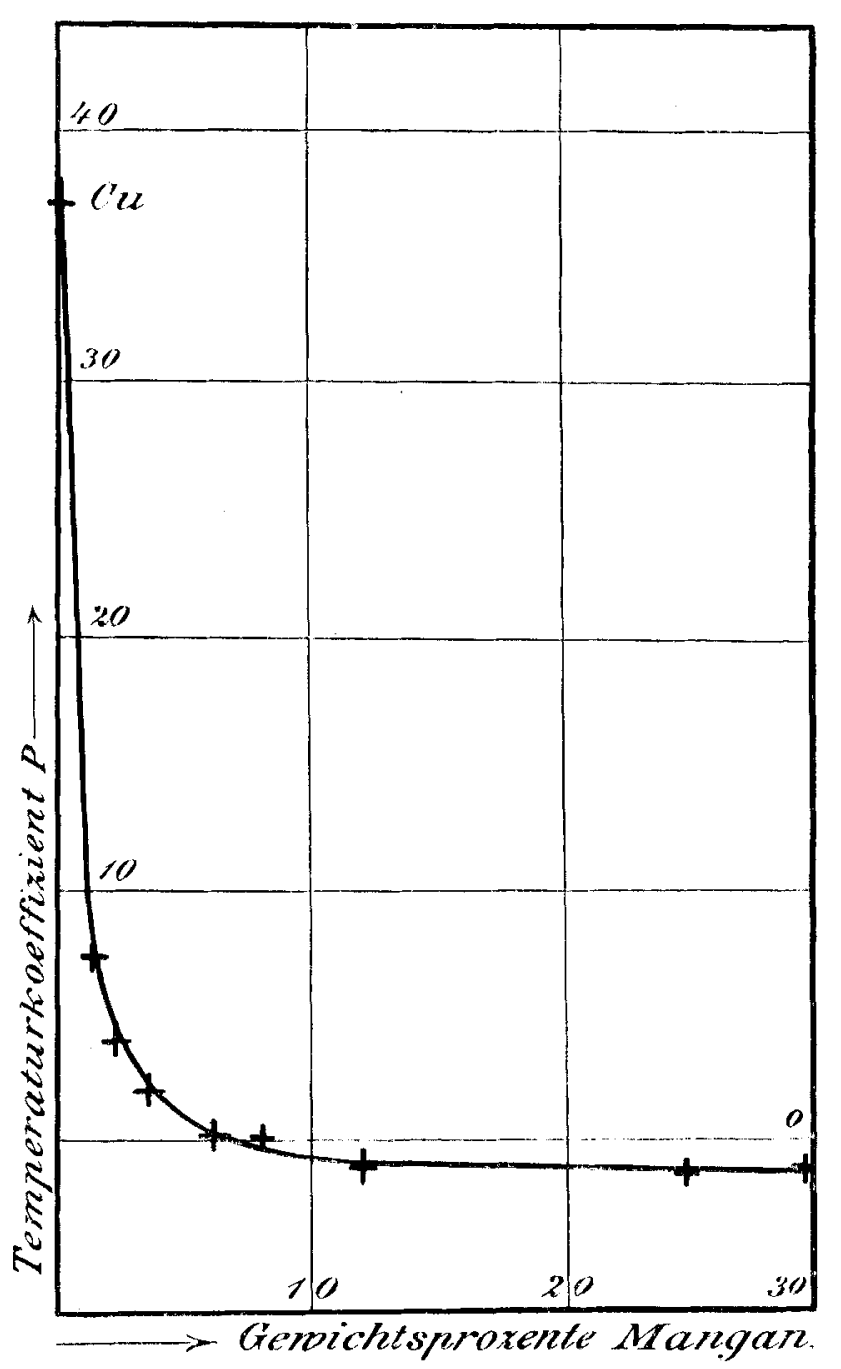

Fig. 6. Kupfer-Mangan nach Feosiner und Lindeck.

allerdings in der Fom $\frac{d W}{d t}$ für eine bestimmte Temperatur (cf. S. 62, Gl. (6) und folgende Zeilen). Da diese Gröfse hier sehr stark von 
der Temperatur abhängig ist, und diese Abhängigkeit nicht genau mitgeteilt wird, so liefs sich hier $P$ nicht berechnen. Es sind darum nur in Figg. $5^{1}$ und 6 die von den Verfassern gegebenen Kurven des Temperaturkoeffizienten $\frac{d W}{d t}$ (bei ihnen in anderer Bezeichnungs. weise $\alpha$ genannt) in Abhängigkeit von der Konzentration, reproduziert worden. Wir erkennen auch hier wieder die typische Kurvenform mit dem steilen Absturz von beiden reinen Metallen her, obgleich die Gröfse $\frac{d W}{d t}$ nicht mit $P$ identisch ist (cf. S. 62, Gl. (6) und folgende Zeilen). Dafs wir es hier in diesen Legierungen tatsächlich mit Mischkrystallreihen zu tun haben, war schon in der früheren Arbeit besprochen (1. c. S. 404 und 427).

Alle diese Beispiele weisen also gemeinsam darauf hin, dals sich auf den Kurven, welche die Abhängigkeit des Temperaturkoeffizienten von der Konzentration der Legierungen darstellen, ebensoscharf die Existenz von Mischkrystallen und die Grenzen etwaiger Mischungslücken ausprägen, wie in den Leitfähigkeitskurven selbst.

Einen vollgültigen Beweis vermögen allerdings diese immerhin noch wenig zahlreichen Beispiele nicht abzugeben. Ein von MatThiessen gegebenes Gesetz gestattet jedoch auch vereinzelte Angaben, von denen noch eine grölsere Reihe vorliegt, als Belege heranzuziehen.

\section{Das Gesetz von Matthiessen.}

Nach MATTHiessen ist ganz allgemein in jeder binären Legierung das Verhältnis der nach der Mischungsregel berechneten Leitfähigkeit $x_{m}$ zu der beobachteten, $x$, gleich dem Verhältris des nach der Mischungsregel berechneten Temperaturkoeffizienten $P_{n} \mathrm{zu}$ dem beobachteten $P$. Dieses empirische Gesetz lälst sich ausdrücken durch die Gleichung:

$$
\frac{\varkappa_{m}}{\varkappa}=\frac{P_{m}}{P}
$$

Da nun $P_{m}$ bei fast allen Metallen nahezu den gleichen Wert $29 \pm 2$ hat, so ergibt sich:

1 Der Wert für reines Nickel (53.4) ist der Untersuchung von Dewar und Fleming entnommen. Der von F. u. L. gegebene Wert bezieht sich offenbar auf unreines Nickel und ist za klein. Eine Analyse ihres Nickels geben die Verf. im Gegensatz zu den Legierungen nicht. 


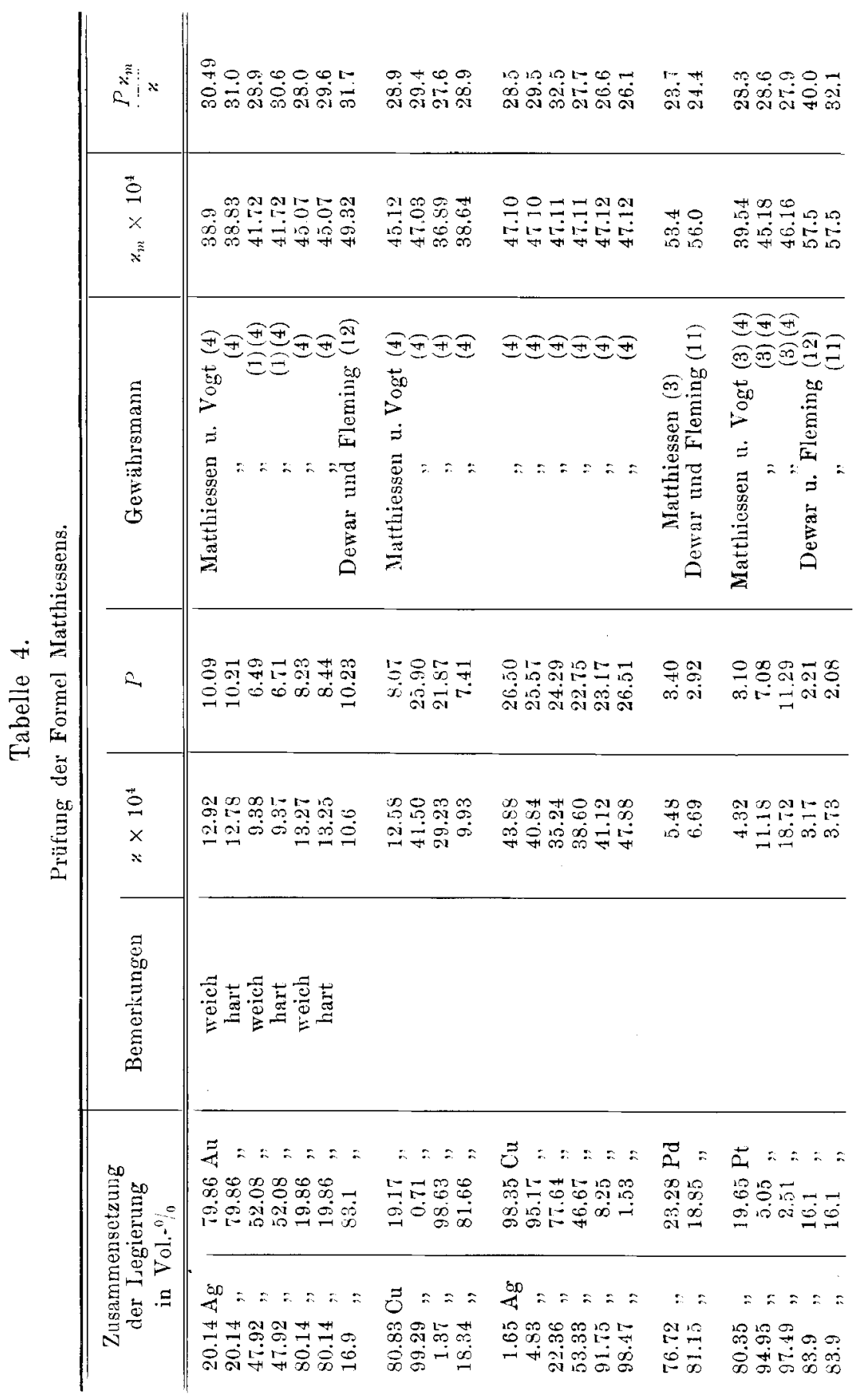




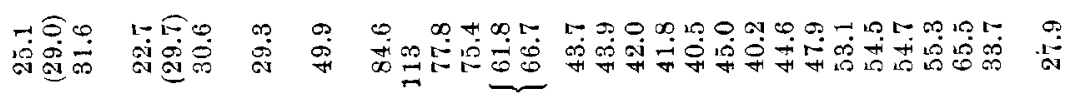

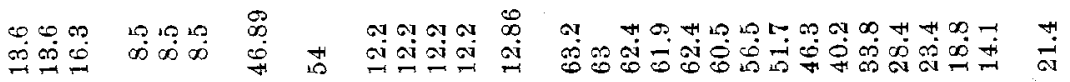

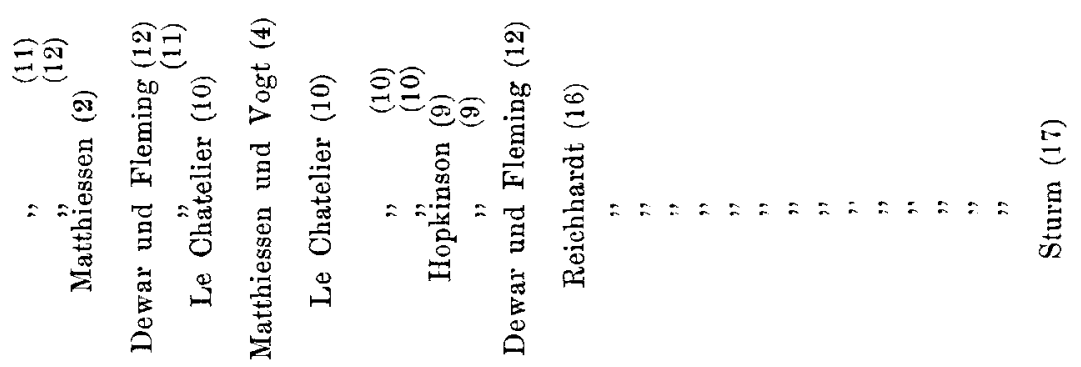

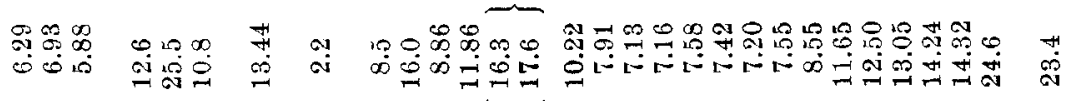

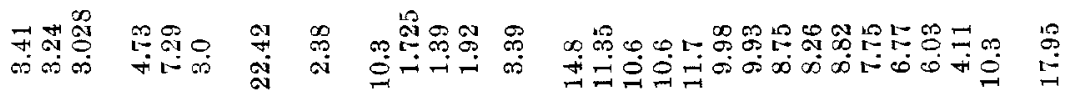

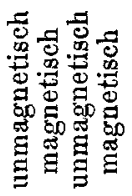

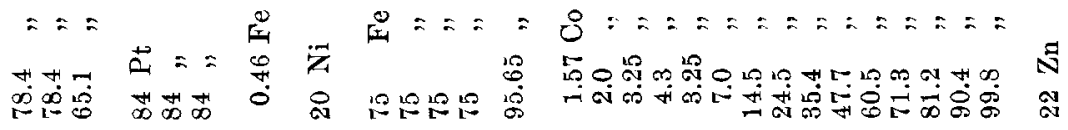

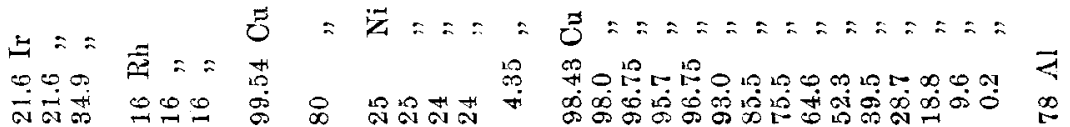




$$
\begin{gathered}
-74- \\
x_{m} P=P_{m}=29 \pm 2,
\end{gathered}
$$

d. h. der Temperaturkoeffizient einer beliebigen Legierung multipliziert mit dem ihm eigenen Quotienten der berechneten und tatsüchlichen Leitfühigkeit mufs immer wieder dieselbe Konstante ergeben.

Die Richtigkeit djeser Beziehung, die Matraiessin selbst schon an einer Reihe von eigenen Messungen bestätigt fand, ist nun auch an den späteren Liten, die wir Dewar und Fleming (11),(12), Le Cinatelier (10), Hopinson (9), Reichuardt (16) und Sturm (17) ${ }^{1}$ verdanken, nachgeprüft worden. Die diesbezüglichen Daten sind in Tab. 4 zusammengefarst. Wir finden in zwei Vertikalkolumnen die beobachteten Werte $x$ und $l^{\prime}$, in einer weiteren $\boldsymbol{x}_{m}$, nach der Mischungsregel berechnet, eingetragen. In der letzten Kolumne sind die Werte angegeben, die sich aus den vorigen nach Gl. (7a) berechnen lassen. Die Werte $x$ und $x_{n i b}$ beziehen sich bei MaTTHHFssen und VOGT (4) auf die T'emperatur von $100^{\circ}$, bei allen anderen auf $0^{\circ}$. REICHAATT gibt den mittleren Temperaturkoeffizieuten von $0-150^{\circ}$ (bei ihm $\alpha$ genannt), so dafs sich $P_{z}$ und weiter $I^{\prime}$ unmittelbar ergaben. Von STuRm(17) (Al-Zn) konnte nur ein Wert berücksichtigt werden, da nur dieser eine Legierung betrifft, die durch sorgfältiges Anlassen in einen stabilen Zustand übergeführt wurde.

Bei Durchsicht der Werte der letzten Kolumne findet man abweichend hohe Werte nur für die Legierungen mit magnetischen Metallen, besonders Eisen-Nickel. Das darf jedoch durchaus nicht wunder nehmen, da für diese Metalle selbst $P 104.1$ und 63.4 (cf. Tab. 1) beträgt. Im übrigen aber ist eine deutliche Konstanz nicht zu verkennen, besonders nicht, wenn man in Betracht zieht, dafs geringe Febler in den Angaben der in aufserordentlich weiten Grenzen schwankenden $P$ - und $x$-Werte und der Konzentrationen grolse Differenzen bei der Berechnung des Endwertes hervorrufen.

Die beiden eingeklammerten Platin-Iridum- und Platin-Rhodiumwerte sind frei angenommen, da die Leitfähigkeiten des reinen Rhodiums und Platins nicht bekannt sind. Daraus ergaben sich die

1 Nicht verwcrten liefsen sich hier einige weitere Daten von Mac Gregor und KNors (6), weil diese Autoren die absoluten Werte der gemessenen Leitfühigkeiten nicht mitteilen. 
Werte der anderen Glieder dieser Legierungen. Die Angaben von Le Chatelifer und Dewar und Fleming bezüglich Mangan-Eisen liefsen sich nicht verwerten, da sie nur eine einzelne Legierung betreffen.

Jedenfalls ergibt sich wohl einwandfrei das Vorhandensein einer Proportionalitit der Erriedrigung von $x$ und $P$, wenn sich auch vielleicht einige Modifizierungen des Gesetzes von Matthiessen späterhin noch ergeben mögen.

$\mathrm{Zu}$ bemerken bleibt, dafs dieses Gesetz auch die mischkrystallfreien Legierungen mit einbegreift. Für diese ist:

folglich nach Gl. (7)

$$
\varkappa_{m}=1,
$$

$$
P=P_{m}=29 \pm 2,
$$

was wir bestätigt fanden.

Sodann stellt Matthiessen in einer Tabelle (l. c. S. 45, Tab. 12) eine Reihe von Legierungen zusammen, die sich seinem Gesetze nicht fügten. Diese Ausnahmen sind jedoch nur scheinbare. Nachdem wir heute wissen, dafs alle diese Legierungen Verbindungen enthalten, ist es selbstverständlich, dafs den Berechnungen von $\boldsymbol{x}_{m}$ und $P_{m}$ die den vorhandenen Verbindungen zukommenden $P$ - und $x$-Werte zugrunde zu legen sind. Matrhiessen, der von der Existenz dieser Verbindungen noch nichts wufste, gelangte daher zu falschen Berechnungen. Hierauf wird noch weiter unten eingegangen werden.

Von Bards (8) sind Einwendungen gegen die Formeln MatTHIESSENs erhoben und andere Beziehungen zwischen der Leitfähigkeit der Legierungen und ihrem Temperaturkoeffizienten aufgestellt worden. Auf diese Kontroverse soll an anderer Stelle eingegangen werden. Hier sei nur angeführt, dafs die Widersprüche zwischen beiden Forschern sich ohne Schwierigkeit beseitigen lassen. Barus greift gewisse Gruppen von Legierungen heraus und es gelingt ihm, für diese gemeinsame Gleichungen aufzustellen, welche von denjenigen Matturessens stark abweichen, ihnen gleichwohl nicht widerstreiten, sondern sogar eine wertvolle Bestätigung derselben ergeben.

\section{Folgerungen.}

Nehmen wir nach dem Vorausgegangenen das Gesetz MATTHIEssens als erwiesen an, so gelangen wir zu dem Schlofs, dals 
nicht allein, wie die weiter oben mitgeteilten Kurven der Temperaturkoeffizienten andeuteten, die $P$-Kurven den $x$-Kurven ähnlich sind, sondern sie stehen sogar in gesetzmälsiger Beziehung zueinander: Die Erniedrigung des Temperaturkoeffizienten ist der Leitfähigkeitserniedrigung gleich.

Die $x_{m}-$ und $P_{m}$-Werte sind dargestellt durch gerade Linien, die die Werte der reinen Komponenten verbinden. Bei Gegenwart von Mischkrystallen tritt in jeder Legierung eine ihr eigentümliche Verkürzung der Ordinaten dieser geraden Linien ein. Die Verkürzung ist stets für die Leitfähigkeit und für den Temperaturkoeffizienten die gleiche. Daher entspricht die tatsächliche Kurve des Temperaturkoeffizienten genau der der Leitfähigkeit selbst.

Nachdem also in der vorangegangenen Arbeit, gesetzmälsige Zusammenhänge zwischen dem Auftreten von Mischkrystallen und der Leitfähigkeit aufgestellt waren, führt der von MATTHIESSEN gegebene Zusammenhang der Leitfähigkeit mit ihrem Temperaturkoeffizienten ohne weiteres zu analogen Beziehungen auch zwischen dem letzteren und der Konstitution.

Diese lassen sich in folgender Weise formulieren:

Die Kurven des Temperaturkoeffizienten der Leitfähigkeit binärer Legierungen, die eine ununterbrochene Reihe von Mischkrystallen bilden, fallen von den Werten der reinen Komponenten zuerst sehr steil ab, und haben gegen die Mitte zu ein sehr flaches Minimum, derart, dafs Zusatz einer gewissen Menge eines zweiten Metalls zum ersten zuerst eine ungleich stärkere Erniedrigung der Leitfähigkeit hervorruft als der gleiche Zusatz bei schon erreichtem höheren Gehalt.

Bei begrenzter Mischbarkeit im krystallisierten $\mathrm{Zu}$ stande entsprechen den Sättigungsonzentrationen zwei Knicke in der Kurvedes Temperaturkoeffizienten. Zwischen den Knicken verläuft die Kurve geradlinig wie bei Gemengen reiner Krystalle.

Durch Einführung des spez. Widerstandes, $W$, statt der Leitfähigkeit, erhält MATTHIESSEN aus Gleichung (7):

$$
P \cdot W=P_{m} \cdot W_{m},
$$


wofür sich nach Gleichung (3) (S. 61) setzen lälst:

$$
\frac{W_{100}-W_{0}}{W_{100}} W=\frac{W_{m 100}-W_{m 0}}{W_{m 100}} W_{m}
$$

Denkt man sich $W$ und $W_{m}$ bei $100^{\circ}$ genommen, so folgt:

$$
W_{100 m}-W_{0 m}=W_{100}-W_{0},
$$

d. h.: die absolute beobachtete Zunahme des Widerstandes mit der Temperatur ist der berechenbaren gleich: was sich auch weiter formulieren lälst: die absolute Zunahme des Widerstandes mit der Temperatur läfst sich für alle binären Legierungen, ob sie Mischkrystalle enthalten oder nicht, annähernd nach der Mischungsregel aus den Werten der reinen Komponenten berechnen:

Ferner läfst sich Gleichung (9) auch schreiben:

$$
W_{100}-W_{100 m}=W_{0}-W_{0 m} .
$$

$W_{100}$ und $W_{0}$ sind darin der gesamte tatsächliche Widerstand der Legierung, $W_{100 m}$ und $W_{0 m}$ derjenige Widerstand, der ihr bei Abwesenheit von Mischkrystallen zukommen würde. Die Differenzen in Gleichung (10) lassen sich also deuten als ein Widerstand, der durch das Vorhandensein von Mischkrystallen hervorgerufen wird und sich dem ursprünglichen zuaddiert. Gl. (10) besagt nun, dals dieser Widerstand von der Temperatur unabhängig ist.

Dieses wichtige Resultat ist in Fig. 7 in räumlichen Koordinaten veranschaulicht. Der Raumkörper, der den nach der Mischungsregel berechenbaren ursprünglichen Widerstand repräsentiert, ist derselbe wie der in

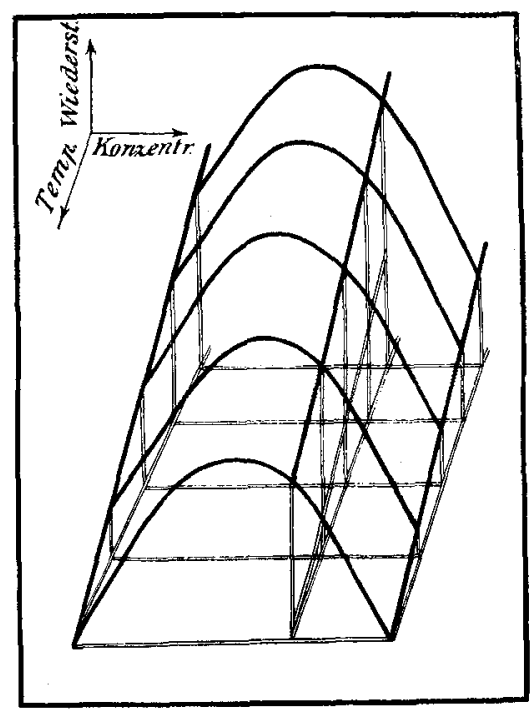

Fig. 7. Widerstand bei Mischkrystallen. Fig. 1 dargestellte. Der bei Mischkrystallen hinzutretende Widerstand überlagert ihn in Form eines 'Tonnengewölbes. 
Hiergegen liefse sich nun der Einwand erheben, dafs dieses Resultat nur durch eine Extrapolation aus den im Intervall von $0^{0}$ bis $100^{\circ}$ gültigen Gesetzmälsigkeiten gewonnen sei, und dafs viel-

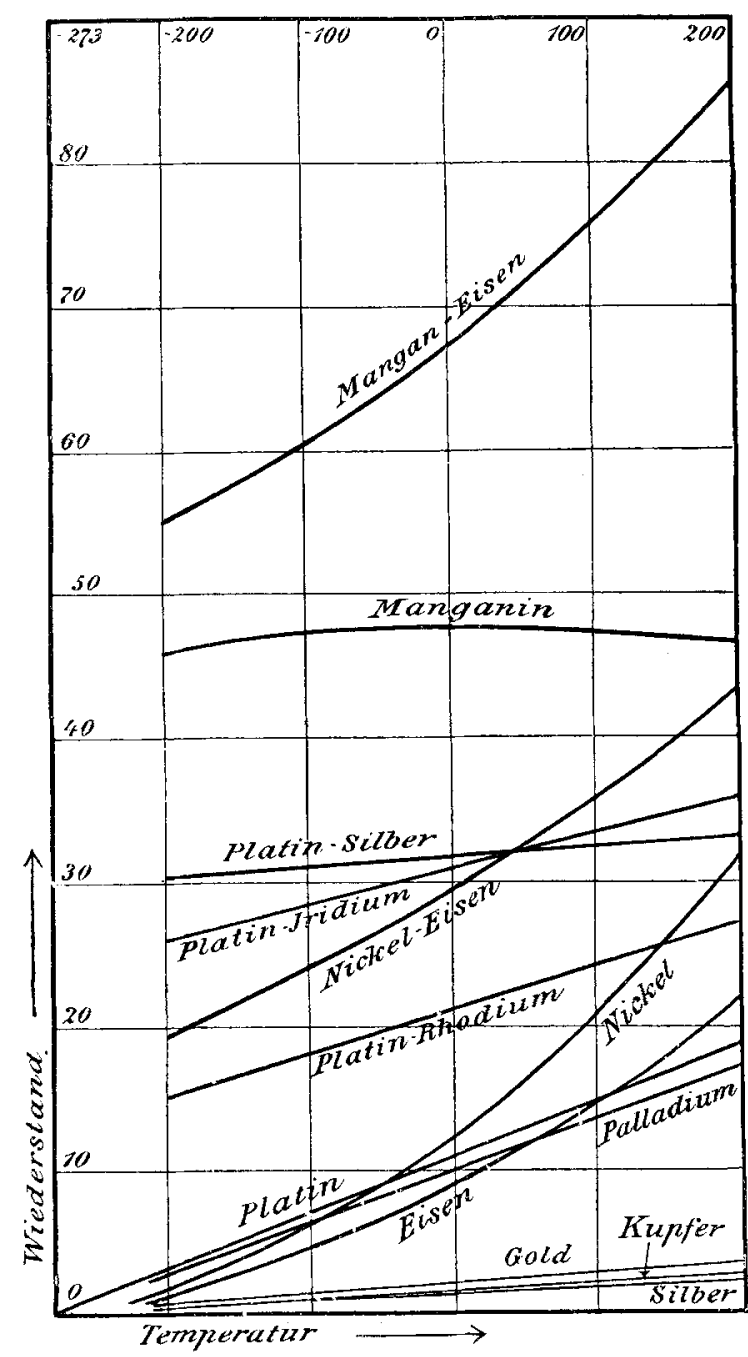

Fig. 8 . leicht die Konstanz von $W-W_{m}$ im Intervall ron $0^{\circ}$ bis $-273^{0}$ nicht bestehen bliebe. Hier lassen sich jedoch die Messungen von Dewar und Fleming (11)(12) als Stichproben anführen, welche bis etwa $-200^{\circ}$ durchgeführt wurden und allgemein ergaben, dals die Konstanz von $W-W_{m}$ in diesem ganzen Temperaturgebiet gewahrt bleibt, obgleich häufig in vielen Fällen $W$ ein Vielfaches von $W_{m}$ betrug. Fig. 8, die einer von den genannten Forschern mitgeteilten Kurventafel entlehnt ist, veranschaulicht wohl deutlich die Unmöglichkeit die experimentell festgelegten Kurven in kontinuierlichem Zuge bei der

Temperatur $-273^{\circ}$ den Widerstand Null erreichen zu lassen.

Das Resultat ist bemerkenswert: Die bei Mischkrystallen beobachtete Erhöhung des Widerstandes kommt nicht dadurch zustande, dal's der ursprüngliche, berechenbare Widerstand durch einen gewissen Faktor vergrölsert erscheint, sondern er addiert sich dem- 
selben zu, zeigt sich durchaus von ihm unabhängig, variiert nicht mit der Temperatur und bleibt auch beim absoluten Nullpunkt unvermindert bestehen, bei welchem den Metallen, aus denen der betr. Mischkrystall besteht, ein Widerstand an sich nicht mehr zukommt. Auf irgendeine Weise ruft also der Umstand an sich, dafs in einem solchen Mischkrystall zwei verschiedene Molekelarten (oder Atomarten) vorhanden sind, einen elektrischen Widerstand hervor. Vielfach ist aus dem Verschwinden des Widerstandes der reinen Metalle beim absoluten Nullpunkt geschlossen worden, dals den Metallen an sich ein Widerstand nicht zukommt, sondern nur dadurch in ihnen erzeugt wird, dafs sie eine bestimmte Temperatur besitzen. Im selben Sinne ruft nach dieser Untersuchung ebenso wie die Temperatur auch innere Inhomogenität in einem Mischkrystall elektrischen Widerstand hervor.

Das in Fig. 8 gegebene Raumdiagramm bedarf für die Fälle begrenzter Mischbarkeit im krystallisierten Zustande nur geringer Modifikation. So wie bei einer gegebenen Temperatur die Sättigungskonzentrationen durch Knicke mit zwischenliegendem geraden Kurvenstück sich ausprägen, so erscheint analog im Raumdiagramm das Tonnengewölbe durch ein flaches Dach abgeplattet, dessen Erhebung über der unteren Schraubenfläche wiederum konstant bleibt. (Ausnahmen treten hier natürlich dann auf, wenn die Sättigungskonzentrationen sich mit der Temperatur verschieben.)

\section{Ausnahmen von Matthiessens Regel.}

Aus der Gleichung

$$
\frac{\varkappa}{\varkappa_{m}}=\frac{P_{a}}{P_{a m}}
$$

folgt, dafs $P_{a}$ und $P_{a m}$ stets das gleiche Vorzeichen besitzen müssen. Da also alle reinen Metalle einen positiven Temperaturkoeffizienten besitzen, dürfte auch bei Legierungen ein negativer Temperaturkoeffizient nicht auftreten.

Nun ist jedoch bekannt, dafs der spez. Widerstand der (allerdings ternären) Manganinlegierungen bei steigender Temperatur ein Maximum erreicht und dann wieder fällt. Dieselbe Erscheinung tritt nach den Untersuchungen von Fudssner und Lindeck (14) bei den Kupfer - Nickel- und Kupfer-Manganlegierungen auf, wie die ihrer Arbeit entnommenen Figg. 5 und 6 zeigen. Besonders deutlich zeigt Fig. 9, welche den spez. Widerstand in Abbängigkeit von 
der Temperatur für mehrere Kupfer-Manganlegierungen gibt, deutlich, dals hier nicht zufällige Unregelmälsigkeiten vorliegen. Die

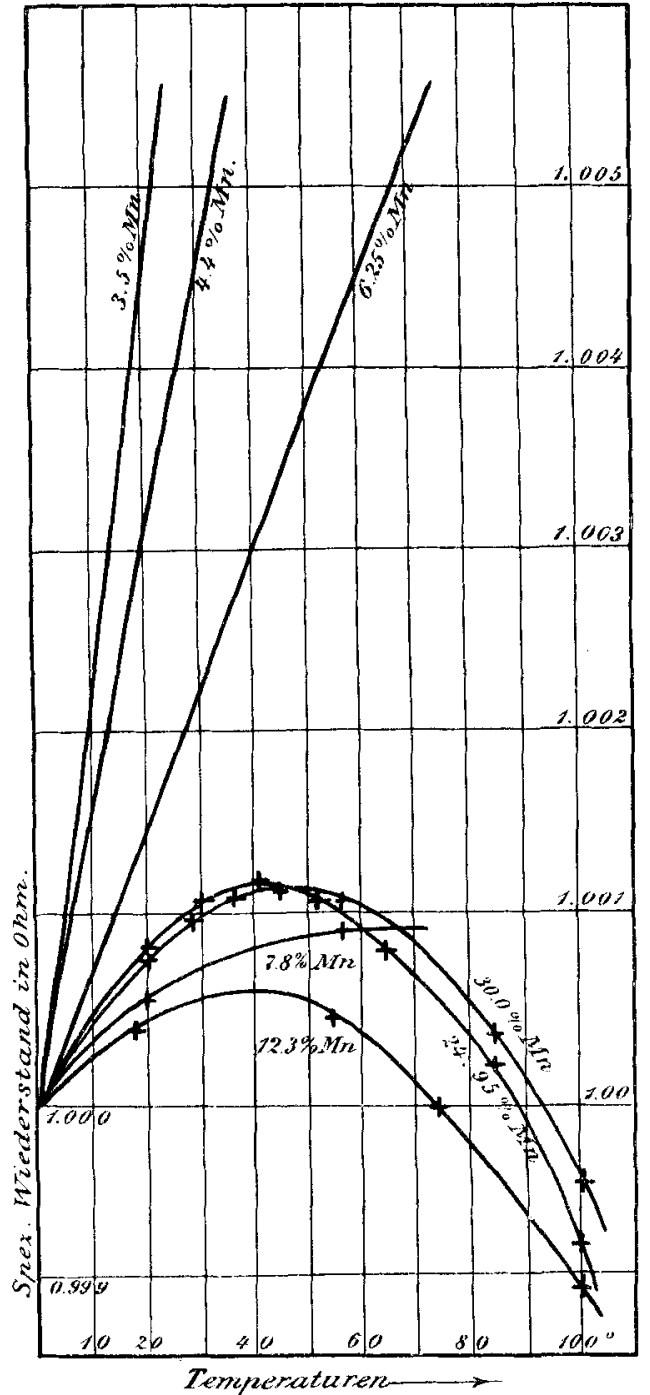

Fig. 9. Widerstand der Kupfer-Manganlegierungen nach Feussner u. Linneck.

Kurven, bei geringem Mangangehalt noch nahezu gerade Linien, gehen mehr und mehr in eine parabolische Gestalt über. ${ }^{1}$

Trotz dieser Abweichung von Mat'THIESSENS Regel ist die typische Form der $P$-Kurven gewahrt geblieben, wie die Figuren 5 und 6 schon zeigten.

Unter den in Fig. 8 wiedergegebenen $W_{-t}$-Kurven nach Dewar und FueMING findet sich eine, die sich auf Manganin bezieht. Auch sie weist ein Maximum, also bei höheren Temperaturen negativen Temperaturkoeffizienten auf. Bei tiefen Temperaturen verläuft sie regelmälsig und den anderen Kurven analog. Es ist wahrscheinlich, dals bei hohen Temperaturen alle Kurven mehr oder weniger Abweichungen von den gegebenen Gesetzen zeigen mögen, ${ }^{1}$ nicht aber bei tiefen, so dafs die wichtigsten Folgerungen allgemeine Gültigkeit behalten.

1 Dic allmählichen Übergänge bei höheren Temperaturen von geraden Linien zu parabolischen Formen erinnert an die analogen Erscheinungen bei den Kurven der Thermokräfte (vergl. z. B. Dewar und Fleming, was vielleicht nicht uninteressant ist in Anbetracht der mannigfachen, zum Teil erfolgreichen Versuche, Thermokräfte und Leitfähigkeit gesetzmälsig zu verknüpfen. 


\section{Legierungen mit Verbindungen.}

Schon bei den Untersuchungen über die Leitfähigkeit konnten die gefundenen Gesetzmälsigkeiten auf diejenigen Legierungen, in denen Verbindungen vorhanden sind, ausgedehnt werden, wenn das Zustandsdiagramm durch Schnitte entsprechend den vorhandenen Verbindungen in Teildiagramme zerlegt gedacht wurde.

Ebenso soll hier verfahren werden.

Um die Regel MatThiessens auch an diesen Fällen erproben zu können, müssen zur Berechnung von $P_{m}$ und $x_{m}$ in jedem Teildiagramm die $P$ - und $x$-Werte, welche den betr. Verbindungen zukommen, zugrunde gelegt werden. Nun aber ist bei den relativ wenigen hierher gehörigen Legierungen der $x$-Wert der in Betracht kommenden Verbindung häufig, der $P$-Wert stets unbekannt.

Es lärst sich nicht a priori sagen, ob der den reinen Metallen zukommende Wert $P=29 \pm 2$ auch den Metallverbindungen eigen ist. Einige Angaben von Matrhiessen, welche sich auf Legierungen von Zinn mit Gold, Silber und Kupfer beziehen, gestatten eine Extrapolation. Wir wissen, durch Untersuchungen von VogEL, Petrenko und Heycock und Neville (cf. die Zitate am Schluls der vorangegangenen Arbeit), dals die Legierungen aus mischkrystallfreien Gemengen ron Zinn mit den Verbindungen $\mathrm{AuSn}_{4}, \mathrm{Ag}_{3} \mathrm{Sn}$ und CuSn bestehen. Setzen wir voraus, dafs auch hier, wie sonst in mischkrystallfreien Legierungen $P$ linear von der Konzentration abhängt, so gelangen wir zu einer Extrapolation des $P$-Wertes der Verbindungen selbst. Nach der Zusammenstellung der erhaltenen Werte in Tabelle 5 scheint der Temperaturkoeffizient der Metall-

Tabelle 5 .

\begin{tabular}{|c|c|c|}
\hline Legierung & Gewährsmann & $P$ \\
\hline Sn & Matthiessen und Vogt & 30.1 \\
\hline 3.48 Vol. $-\% A g$ & , & 30.00 \\
\hline $24.49 \mathrm{SnAg}_{3}$ & (extrapoliert) & $\begin{array}{l}29.18 \\
26.8\end{array}$ \\
\hline 6.43 Vol. $-\% \mathrm{Cu}$ & Matthiessen und Vogt & 28.71 \\
\hline $16.4 \mathrm{CuSn} \quad "$ & (extrapoliert) & $\begin{array}{l}26.24 \\
22.0\end{array}$ \\
\hline 9.68 Vol. $-0 \% \mathrm{Au}$ & Matthiessen und Vogt & 24.20 \\
\hline $20.46 \underset{\mathrm{AuSn}_{4}}{\#} "$ & $\begin{array}{c}" \\
\text { (extrapoliert) }\end{array}$ & $\begin{array}{l}22.90 \\
22.4\end{array}$ \\
\hline
\end{tabular}


verbindungen, wenn auch nicht sebr viel, so doch deutlich geringer zu sein, als der der reinen Metalle. Der spez. Widerstand der Verbindungen verschwindet beim absoluten Nullpunkt nicht, und wir können sagen, dafs: Ebenso wie den metallischen Mischkrystallen ein spez. Mischkrystallwiderstand zukommt, so auch ganz analog den Metallverbindungen ein spez. "Verbindungswiderstand" zukommt, der den Komponenten an sich nicht eigen ist, sondern in der Verbindung dadurch hervorgerufen wird, dals verschiedene Atome im Molekül vorhanden sind.

Einige weitere Daten betr. Legierungen, die Verbindungen enthalten, wie Silber-Blei, Silber-Aluminium, Kupfer-Aluminium, TitanAluminium, Arsen-Kupfer, Phosphor-Kupfer, Zinn-Kupfer, EisenSilicium, Eisen-Gold [cf. Dewar (11) (12), Weber (7), Matthiessen (4), Le Chatelier (10)], sind vorerst nicht verwertbar, da diese zugleich Mischkrystalle enthalten und die nötigen weiteren Daten bezüglich der Formeln der betreffenden Verbindungen und ihrer $P$ - und $x$-Werte noch fehlen.

Sehr zahlreiche Daten hingegen besitzen wir bezüglich der Kupfer-Zinklegierungen mit $0-50 \% \mathrm{Zn}$ durch MatThiEsSEN und VOGT (4) und HaAS (13). ${ }^{1}$ Aus der Leitfähigkeitskurve ergab sich in der früheren Arbeit (1. c. S. 419 und 420), dals die von 47 bis etwa $50 \%$ Zink sich erstreckende Mischkrystallreihe, die durch SHEPheard mit Hilfe der mikrographischen Analyse nachgewiesen wurde, eine Verbindung enthalten mufs. Wenn dieser eine einfache Formel zukommt, so kann dieselbe nur CuZn sein. Demnach sind die untersuchten Kupfer-Zinklegierungen mit weniger als $50 \% \mathrm{Zn}$ als Legierungen von Kupfer mit der Verbindung CuZn aufgefafst und die mitgeteilten Gewichts- und Atomprozente in Volumprozente $\mathrm{CuZn}$ umgerechnet worden mit Hilfe der von MAEY (Zeitschr. f. physikal. Chem. 38 (01) 289, 292) mitgeteilten spez. Gewichte. In Tabelle 6 sind die Volumprozente CuZn mit den zugehörigen Werten des Temperaturkoeffizienten vereinigt und in Fig. 10 graphisch dargestellt.

Die durch die Punkte hindurchgelegte Kurve zeigt einen deutlichen Knick bei 76 Volumprozenten CuZn. Bei dieser Konzentration fand in der Tat Shepheard die Mischungsgrenze einer von hier bis zum Kupfer sich erstreckenden Mischkrystallreihe. Die Kurve des Temperaturkoeffizienten fällt in diesem Intervall vom

1 Angaben von Le Chatelier (10), Thle (15) und Bewoit (5) stehen vereinzelt und sind darum hier nicht berücksichtigt. 
Tabelle 6 .

\begin{tabular}{|c|c|c|c|c|c|c|c|}
\hline 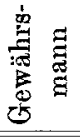 & $\begin{array}{c}\mathrm{I} \\
\text { Gew.- } \% \\
\mathrm{Zn}\end{array}$ & $\begin{array}{c}\text { II } \\
\text { Vol. } . \% \\
\text { CuZn }\end{array}$ & $\begin{array}{l}\text { III } \\
P\end{array}$ & $\begin{array}{l}\text { IV } \\
P_{m}\end{array}$ & $\begin{array}{c}\mathrm{V} \\
x_{m}(W \text { linear } \\
\text { angenommen })\end{array}$ & $\begin{array}{l}\text { VI } \\
x\end{array}$ & $\begin{array}{c}\text { VII } \\
P \text { ber. }\end{array}$ \\
\hline \multirow{15}{*}{ 急 } & 0 & 0 & 30.2 & 30.2 & 63.1 & 63.1 & 30.2 \\
\hline & 0.71 & 1.53 & 27.1 & 30.23 & 62.0 & 54.56 & 26.6 \\
\hline & 1.56 & 3.42 & 24.1 & 30.265 & 60.8 & 46.88 & 23.3 \\
\hline & 3.07 & 6.59 & 22.6 & 30.33 & 58.8 & 42.16 & 21.75 \\
\hline & 5.51 & 11.8 & 19.25 & 30.43 & 55.8 & 33.22 & 18.1 \\
\hline & 9.08 & 19.3 & 16.9 & 30.58 & 52.0 & 27.49 & 16.2 \\
\hline & 18.02 & 37.7 & 14.48 & 30.95 & 44.6 & 21.00 & 14.6 \\
\hline & 20.29 & 42.3 & 14.08 & 31.06 & 43.0 & 19.75 & 14.3 \\
\hline & 22.71 & 47.0 & 13.86 & 31.15 & 41.5 & 18.44 & 13.85 \\
\hline & 28.16 & 57.8 & 13.67 & 31.37 & 38.5 & 17.16 & 14.0 \\
\hline & 34.23 & 69.5 & 13.64 & 31.63 & 35.7 & 15.87 & 14.05 \\
\hline & 40.28 & 80.9 & 17.48 & 31.88 & 33.3 & 17.27 & 16.6 \\
\hline & 42.55 & 85.1 & 19.23 & 31.97 & 32.5 & 18.84 & 18.4 \\
\hline & 45.19 & 89.9 & 22.17 & 32.08 & 31.7 & 21.22 & 21.5 \\
\hline & $\begin{array}{c}46.85 \\
\text { Vol.- } \% \mathrm{Zn}\end{array}$ & 92.7 & 23.7 & 32.15 & 31.2 & 23.18 & 23.9 \\
\hline \multirow{5}{*}{ 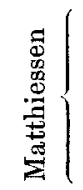 } & 5.03 & 8.89 & 20.61 & 29.4 & 57.5 & 40.252 & 20.6 \\
\hline & 10.88 & 22.83 & 17.41 & 28.1 & 50.4 & 31.289 & 19.0 \\
\hline & 23.61 & 42.2 & 12.80 & 28.8 & 43.0 & 14.200 & 10.3 \\
\hline & 29.45 & 52.5 & 11.49 & 31.43 & 40.0 & 14.472 & 11.4 \\
\hline & 42.06 & 75.3 & 12.40 & 29.4 & 34.5 & 14.528 & 12.4 \\
\hline
\end{tabular}

Kupfer steil ab. Bei höheren Zinkkonzentrationen entspricht der Mischungslücke ein geradliniges Stück der $P$-Kurve. Bei mehr als $93 \% \mathrm{CuZn}$ ist dann wieder ein steilerer Anstieg der Kurve zu erwarten (etwa wie in der Figur punktiert angedeutet), doch fehlen hier die Messungen.

In diesem Beispiel entspricht also auch bei Gegenwart einer Verbindung die Gestalt der $P$-Kurve den oben gegebenen allgemeinen Regeln.

$\mathrm{Zu}$ prüfen bleibt, ob auch darüber hinaus die von MATTHIESSEN gegebene quantitative Beziehung zur Leitfähigkeit selber gültig bleibt. $\mathrm{Zu}$ diesem $\mathrm{Zwecke}$ ist die Leitfähigkeit der Verbindung CuZn gemäls Fig. 17 in der vorangegangenen Arbeit mit Hilfe einer geringen Extrapolation zu $30 \times 10^{-4}$ und der Temperaturkoeffizient derselben versuchsweise zu 31 angenommen worden. Mit Hilfe dieser Werte sind für die einzelnen Legierungen $P_{m}$ und $x_{m}$ berechnet und in Kolumne IV und $V$ der Tabelle 6 zusammengestellt. 
Die Kolumne VI enthält aufserdem die Leitfähigkeitswerte derselben Legierungen, wie sie von den Verfassern mitgeteilt sind, und schon in der vorangegangenen Arbeit zur Konstruktion der Leit-

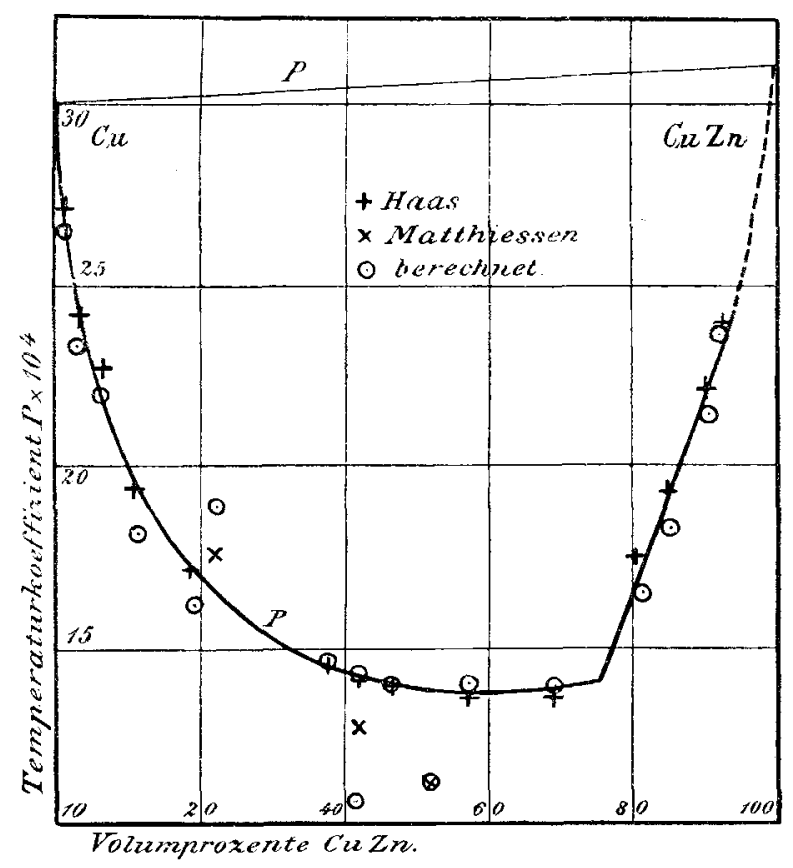

Fig. 10. Die Legierungen $\mathrm{Cu}-\mathrm{CuZn}$.

fähigkeitskurve dienten. Aus diesen Werten wurden durch Multiplikation mit dem Quotienten $\stackrel{P}{m}_{\varkappa_{m}}$ gemäls einer Umformung von Mattulesseaxs Gleichung:

$$
P=x \frac{P_{m}}{\varkappa_{i n}}
$$

$P$ berechnet. Die Resultate sind in Kolumne VII gegeben und in Fig. 10 als kleine Kreise eingetragen. Es zeigt sich, dals sie sich gleichfalls der Kurve gut anschliefsen, dafs hier also Matthiessens Regel auch bei der Anwendung auf Legierungen mit Verbindungen sich bewährt.

\section{Zusammenfassung.}

Die von MatThIEssen gegebenen Beziehungen zwischen der Leitfübigkeit und ihrem Temperaturkoeffizienten werden durch die späteren Messungen anderer Autoren bestätigt. Bei sinnentsprechender Erweiterung lälst sich das Gesetz sogar auf diejenigen 
Legierungen anwenden, die Verbindungen enthalten. Dadurch kïären sich Ausnahmen auf, die MatThiessen selbst von seinem Gesetz machen zu müssen glaubte, und fallen fort.

Ausnahmen bilden nur einige Legierungen mit negativen Temperaturkoeffizienten (cf. S. 79). Die charakteristische allgemeine Gestalt der Kurven des Temperaturkoeffizienten in Abhängigkeit von der Zusammensetzung bleibt jedoch auch hier gewahrt.

Ganz allgemein läfst sich sagen: Es besteht mit wenigen Ausnahmen Proportionalität $z$ wischen den Leitfähigkeiten der verschiedenen Legierungen zweier Komponenten und den zugehörigen Temperaturkoeffizienten, a uch dann, wenn Mischkrystalle und Verbindungen in beliebiger Ausdehnung und Anzahl vorliegen, und ferner: Ausnahmslos entspricht die Gestalt der Kurven des Temperaturkoeffizienten genáu der Gestalt der Kurven der Leitfähigkeit.

Der den Rechnungen zugrunde gelegte Ausdruck $P$ bedeutet allerdings nur ein Provisorium. Das gewählte Temperaturintervall von $0-100^{\circ}$ war beliebig, ebenso wie die Wahl von $P_{a}$ statt $P_{z}$ (cf. S. 4). Bei genauerem Studium der vorliegenden Regeln dürften $\alpha$ und $\beta$ gesondert zu betrachten sein.

Durch diese Gesetzmälsigkeiten gelangen wir von den früher gegebenen Zusammenbängen zwischen der Konstitution und der Leitfähigkeit der Legierungen $\mathrm{zu}$ solchen auch zwischen der Konstitution und dem Temperaturkoeffizienten der Leitfähigkeit. Aus den Kurven der Temperaturkoeffizienten lassen sich ebenso entsprechend den Knicken die Konzentrationen der gesättigten Mischkrystalle und der Verbindungen, aus dem geradlinigen oder stark gekrümmten Verlauf der einzelnen Kurvenstücke das Vorhandensein zweier oder nur einer Krystallart erkennen, wie aus den Kurven der Leitfähig keiten selbst.

Dieses Ergebnis besitzt eine wertrolle praktische Anwendung:

Die direkten Methoden der Widerstandsmessung sind häufig nicht anwendbar, weil die Sprödigkeit vieler Legierungen das Ausziehen von Drähten nicht gestattet, auch die Herstellung von gröfseren gegossenen Stäßsen ohne Saigerungen, Risse usw. nicht gelingt. Auch die indirekten Methoden versagen nur allzuoft bei den brüchigen Legierungen, ${ }^{1}$ bei denen die Herstellung kleiner

1 Gerade die Metallverbindungen sind allgemein durch höhere Sprödigkeit ausgezeichnet. 
Stückchen von exakt definierten mechanischen Dimensionen unmöglich ist.

Nunmehr genügt es jedoch, unter Verzicht auf den absoluten Betrag der Leitfühigkeit einer Legierung, nur deren Temperaturkoeffizienten $P$ experimentell festzustellen. Zwei Widerstandsmessungen in beliebigem Mafse bei $0^{\circ}$ und $100^{\circ}$ liefern nach der Gleichung:

$$
P=\frac{W_{100}-W_{0}}{W_{100}}
$$

die Werte von $P$, und die aus diesen gebildete Kurve gibt die gesuchten Aufschlüsse über die Konstitution der betr. Legierungsreihe. Diese Messungen werden durch Vorhandensein von Luftblasen, Krystallisationshohlräumen usw. nicht beeinträchtigt. Bei Verwendung indirekter Methoden (Wirbelströme, Dämpfung der Schwingungen im Magnetfeld usw.) ist sogar die Verwendung der Reguli in beliebiger Form als Pulver, Reguli, einzelne kleine Splitter, Krystalle oder Drahtstückchen möglich.

Von theoretischen Gesichtspunkten aus beansprucht wohl das meiste Interesse das Ergebnis, dafs der in Mischkrystallen durch die Gegenwart ungleichartiger Molekeln (resp. Atome) hervorgerufene Widerstand keinerlei Abhängigkeit von dem ursprünglichen Widerstando der betr. Legierung, wie er sich nach der Mischungsregel ergibt, zeigt, sondern unabhängig von der Temperatur auch beim absoluten Nullpunkt unvermindert besteht, obwohl den Komponenten des Mischkrystalles an sich ein Widerstand bei dieser Temperatur nicht mehr zukommt.

Es sind von Kohlrausch, Weber, Lorenz, Ostwald, Raylergh und endlich Lrebenow Versuche gemacht worden, den Leitungswiderstand der Metalle und die unerwarteten starken Vermehrungen desselben in manchen Legierungen auf Thermokräfte zwischen den Komponenten zurückzuführen. Diese Versuche erfahren vielleicht neve Beleuchtung durch die Erkenntnis, dafs solche starke Widerstandserhöhungen gerade den Mischkrystallen und nur diesen eigen sind und beim absoluten Nullpunkt der Temperatur unvermindert bestehen bleiben.

\section{Literatur.}

1) Matrhieseen 1861, Pogg. Ann. 112, 353.

2) Matthiessex 1862, Report. Brit. Assoc. (62) 136.

3) Matrhiessrn 1863, Report. Brit. Assoc. (63) 127.

4) Matruessen und Vogt 1864, Pogg. Ann. 122 (64) 19. 
5) Benort 1873, Compt. rend. 76, 342.

6) Mac. Gregor und Kxott 1880, Trans. Roy. Soc. Edind. 29, 099.

7) Weiser 1888, Wied. Ann. 34, 576.

8) Barus 1888, Sill. Amer. Journ. Ścience [3] 36, 427.

9) Hopkinson 1890, Proc. Roy. Soc. Lond. 47, 138.

10) Le Chatelier 1890, Compt. rend. 110, 283; 111, 454.

1894, Compt. rend. 119, 272.

1901, Contribution à l'étude des alliages (Paris) 413.

11) Dewar und Fleming 1892, Phil. Mag. [5] 34, 326.

12) Dewar und Fleming 1893, Phil. Mag. [5] 36, 271.

13) H HaS 1894, Wied. Ann. 52, 673.

14) Feussner u. Lindeck 1895. Abhandign. d. phys.-techn. Reichsanstalt 2, 501.

15) Ihte 1896, Jahresber. d. kgl. Gymn. Dresden-Neustadt 22, 3.

16) ReichHard' 1901, Drudes Ann. 6, 832.

17) Sтunм 1904, Dissertation, Rostock.

18) Liebenow 1897, Z. f. Elehtrochemie 4, 201.

19) Guertler 1906, Z. anorg. Chem. 51, 397.

\section{Notiz.}

Zur ersten Arbeit (Z. anorg. Chem. 51 (06), 397).

Herr Dr. Voges war so liebenswürdig, mich darauf aufmerksam zu machen, dafs in den Arbeiten Matrhiessens, auf die ich in meinen Arbeiten häufig Bezug nahm, die in den Tabellen mitge-

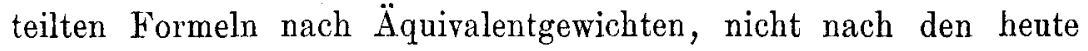
üblichen Atomgewichten zu verstehen seien, wie sich durch Vergleich der Formeln mit den gleichfalls mitgeteilten Volumprozenten ergab.

Da ich nun bei Verwertung der Angaben Matthiessens zwar im allgemeinen die mitgeteilten Volumprozente übernahm, bei den Gold-Zinn und Gold-Bleilegierungen, wegen der in diesen auftretenden Verbindungen, jedoch die Formeln, so bedürfen die Angaben über diese Legierungen einer Korrektur. Fig. 13 ist durch eine andere zu ersetzen, die nebenstehend mitgeteilt ist. Das Maximum in der Leitfähigkeitskurve entspricht nunmehr der Verbindung AuSn. Der Knick, der bislang bei der Formel AuSn lag, rückt nach $\mathrm{Au}_{2} \mathrm{Sn}$, entspricht also nicht mehr einer Verbindung und dürfte, da er ohnehin nur äufserst schwach angedeutet ist, ohne den Melsresultaten Matruiessens Zwang anzutun, in Fortfall kommen. In den Konzentrationsintervallen $\mathrm{AuSn}-\mathrm{AuSn}_{2}$ und $\mathrm{AuSn}_{4}-\mathrm{Sn}$ finden wir auch jetzt wieder geradlinigen Verlauf. $Z$ wei Knicke finden wir bei den Verbindungen $\mathrm{AuSn}_{2}$ und $\mathrm{AuSn}_{3}$ statt $\mathrm{AuSn}_{2}$ und $\mathrm{AuSn}_{4}$. Diese Unregelmälsigkeiten finden vermutlich auch hier wieder durch die von VOGEL nachgewiesenen Umhüllungen ihre Erklärung. 


\section{$-88-$}

Betreffend der Gold-Pleilegierungen ist zu sagen, dafs dem Minimum der Leitfähigkeitskurve, aus welchem auf die Existenz

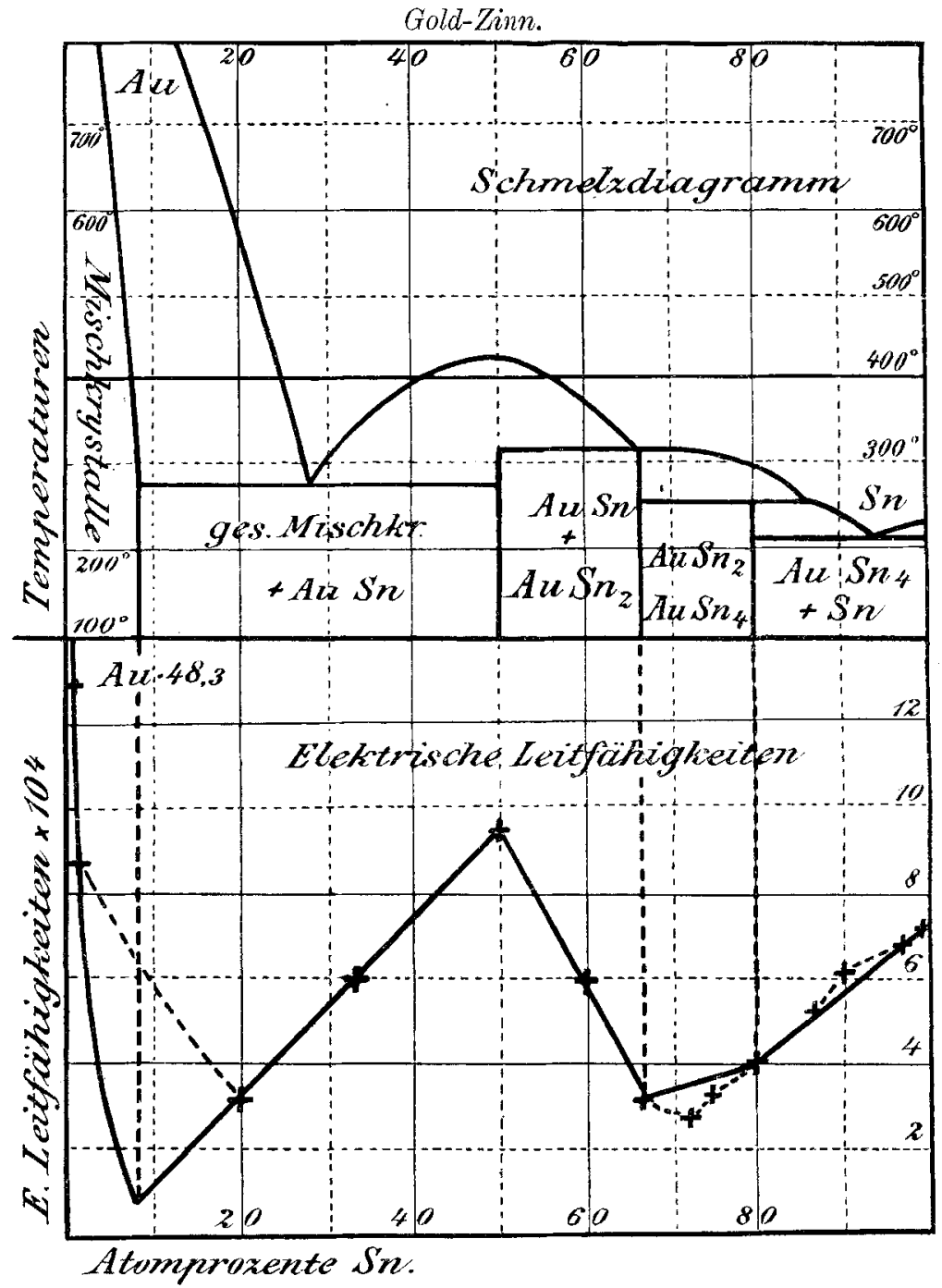

Fig. 13.

einer Verbindung in dem nach Vogen selrr rasch sich oxydierenden Eutektikum geschlossen wurde, der Formel $\mathrm{AuSn}_{3}$, nicht $\mathrm{AuSn}_{6}$, entspricht (cf. S. 415 oben).

Gättingen, Institut für anorganische Chomie der Universität.

Bei der Redaktion eingegangen am 24. März 1907. 